\title{
Insegurança alimentar e fatores sociais, econômicos e nutricionais em estudantes de escolas rurais
}

\section{Food insecurity and the relationship with social, economic and nutritional factors in rural school students}

\author{
Marilene Cassel Bueno' (D), Jaquieli Gruhm Franco², Greisse Viero da Silva Leal³, \\ Vanessa Ramos Kirsten ${ }^{3}$ (D) \\ 'Programa de Pós-graduação em Desenvolvimento Rural, Universidade Federal do Rio Grande do Sul (UFRGS) - Porto Alegre (RS), \\ Brasil. \\ 22Programa de Residência Multiprofissional Integrada em Vigilância em Saúde, Universidade Federal de Santa Maria (UFSM) - \\ Santa Maria (RS), Brasil. \\ ${ }^{3}$ Departamento de Alimentos e Nutrição, Universidade Federal de Santa Maria (UFSM) - Palmeira das Missões (RS), Brasil.
}

Como citar: Bueno MC, Franco JG, Leal GVS, Kirsten VR. Insegurança alimentar e fatores sociais, econômicos e nutricionais em estudantes de escolas rurais. Cad Saúde Colet, 2021;29(2):153-162. https://doi.org/10.1590/1414-462X202129020204

\begin{abstract}
Resumo
Introdução: Segurança alimentar é definida por lei e refere-se ao acesso a alimentos de qualidade e em quantidade suficiente. Objetivo: Avaliar a insegurança alimentar e sua relação com a classe econômica, o programa de transferência de renda e o estado nutricional de estudantes de escolas rurais. Método: Estudo transversal, com estudantes de escolas rurais, que utilizou a Escala Brasileira de Insegurança Alimentar e a classificação socioeconômica da Associação Brasileira de Empresas de Pesquisa. O estado nutricional foi avaliado por meio do Índice de Massa Corporal e do índice Estatura para Idade. Resultados: Foram avaliadas 121 famílias com 157 crianças e adolescentes, das quais 57,9\% se encontravam em insegurança alimentar e 46,3\% eram beneficiárias do Programa Bolsa Família. As famílias de classes socioeconômicas mais baixas estiveram relacionadas com a insegurança alimentar $(p<0,0001)$. As famílias participantes do Programa Bolsa Família obtiveram maior prevalência de insegurança alimentar ( $p<0,0001$ ). Ao avaliar o estado nutricional, foram encontrados altos percentuais de obesidade em crianças (19,7\%) e adolescentes (22,9\%). Conclusão: As famílias rurais avaliadas apresentaram alta prevalência de insegurança alimentar relacionada com a menor classe socioeconômica e a participação do Programa Bolsa Família. Palavras-chave: segurança alimentar; população rural; estado nutricional; condições socioeconômicas.
\end{abstract}

\begin{abstract}
Background: Food security is defined by law, and refers to access to quality food in enough quantity. Objective: To evaluate food insecurity and its relation to the economic class, income transfer program and nutritional status of rural school students. Method: A cross-sectional study conducted with rural school students using the Brazilian Food Insecurity Measurement Scale and the socioeconomic classification of the Brazilian Association of Research Companies. Nutritional status was evaluated through the Body Mass and Height Index according to age. Results: A total of 121 families with 157 children and adolescents were evaluated. Of these, $57.9 \%$ were under food insecurity and $46.3 \%$ were beneficiaries of the Bolsa Familia Program. The lowest socioeconomic classes were associated with food insecurity $(p<0.0001)$. Families
\end{abstract}

Trabalho realizado nas Escolas Municipais da zona rural - Palmeira das Missões (RS), Brasil.

Correspondência: Marilene Cassel Bueno. E-mail: marilenecassel@outlook.com

Fonte de financiamento: nenhuma.

Conflito de interesses: nada a declarar.

Recebido em: Maio 25, 2018. Aprovado em: Mar. 11, 2020
Este é um artigo publicado em acesso aberto (Open Access) sob a licença Creative Commons distribuição e reprodução em qualquer meio, sem restrições desde que o trabalho original seja corretamente citado. 
participating in the Bolsa Família Program presented higher prevalence of food insecurity $(p<0.0001)$. Regarding nutritional status, high obesity rates were found in children (19.7\%) and adolescents (22.9\%). Conclusion: The rural families evaluated presented high prevalence of food insecurity associated with lower socioeconomic class and participation in the Bolsa Família Program.

Keywords: food insecurity; rural population; nutritional status; socioeconomic conditions.

\section{INTRODUÇÃO}

A alimentação é um direito humano fundamental, e cabe ao Estado assegurar a permanência das políticas públicas de alimentação. Nessa perspectiva, a segurança alimentar refere-se ao acesso a alimentos de qualidade e em quantidade suficiente sem comprometer outras necessidades básicas, fundamentada nos princípios de promoção de saúde, sustentabilidade e valorização da cultura'.

A implementação de políticas estruturantes, como o fortalecimento da agricultura familiar, em paralelo a programas de transferência de renda, como o Programa Bolsa Família (PBF), tem sido uma abordagem exitosa na diminuição da fome no Brasil'2. Segundo a Pesquisa Nacional por Amostra de Domicílios (PNAD), realizada em 2013, cerca de 52 milhões de brasileiros não têm acesso diário à comida de qualidade e em quantidade suficiente 3 .

Na PNAD, a insegurança alimentar (IA) esteve presente em maior proporção nas áreas rurais do que nas urbanas. Aspectos relacionados ao perfil socioeconômico, como baixa renda e pouca escolaridade, são fatores que determinam a segurança alimentar e nutricional dessas famílias $s^{3,4}$. Vianna e Segall-Corrêa ${ }^{5}$ destacam também que a dificuldade de acesso aos locais de venda de alimentos e aos serviços públicos é um fator relevante para a IA nessas áreas. É na área rural em que se concentra a maior parte da população sem acesso aos serviços públicos².

Além disso, o estudo nacional demonstrou que a IA foi mais prevalente em famílias com moradores menores de 18 anos em relação às constituídas apenas por adultos ${ }^{3}$. Crianças e adolescentes são mais frágeis às condições de $I A$, visto que estão em um período de crescimento e desenvolvimento ${ }^{6}$, e a alimentação adequada é um fator importante para a saúde e a manutenção do estado nutricional dessa população. Pesquisas recentes têm evidenciado um expressivo aumento nos percentuais de obesidade, que atinge todas as faixas etárias, inclusive crianças e adolescentes, favorecendo o desenvolvimento de outras doenças crônicas precocemente? 2 .

Ao considerar a população rural mais vulnerável às condições de $I A$, principalmente as famílias constituídas por menores de 18 anos, o presente estudo teve o objetivo de identificar a prevalência de IA e fatores sociais, econômicos e nutricionais em estudantes de escolas rurais.

\section{MÉTODO}

Foi realizado um estudo transversal no município de Palmeira das Missões, no Rio Grande do Sul, localizado a $372 \mathrm{~km}$ da capital, Porto Alegre, e com um Índice de Desenvolvimento Humano Municipal (IDHM) de 0,737. Pelo censo do IBGE de 2010, conta com uma população estimada de 34.907 moradores, dos quais 4.497 residem na área rural'?

A amostra foi constituída por escolares (crianças e adolescentes) matriculados nas sete escolas municipais localizadas na área rural do município e suas respectivas famílias, representadas pelos pais ou responsáveis. Foram excluídos do estudo os alunos que estudavam nas escolas rurais, mas que residiam na área urbana, e as crianças menores de 5 anos.

Para o cálculo amostral, foram considerados o número de 576 alunos matriculados nas escolas rurais do município no ano de 2016, o percentual máximo de $15 \%$ de IA esperado para a área rural na região Sul do país (de acordo com a PNAD 2013) e um erro amostral de 5\% com nível de confiança de $95 \%$, resultando em um total de 147 alunos.

A coleta de dados foi realizada nas escolas, nos dias de reuniões com os pais ou responsáveis, no período de setembro a novembro de 2016. Os dados foram coletados por meio de questionários para avaliar as condições de IA e perfil socioeconômico da família, 
respondidos por meio de entrevista. Após, foi realizada a avaliação antropométrica dos alunos, em que os pais e responsáveis autorizaram a participação na pesquisa.

A avaliação da IA foi feita por meio da Escala Brasileira de Insegurança Alimentar (EBIA), que permite avaliar a percepção de segurança alimentar da família. Ela é composta por 14 perguntas relacionadas à alimentação, e a pontuação atribuída para esse instrumento é de um ponto para cada resposta "sim". O somatório desses pontos permite classificar as famílias em quatro categorias:

I - Segurança alimentar (zero ponto): a família tem acesso à alimentação de qualidade em quantidade suficiente sem comprometer outras necessidades;

II - Insegurança leve (1 a 5 pontos): revela preocupação da família com o acesso aos alimentos no futuro e a qualidade inadequada de alimentação;

III - Insegurança moderada (6 a 9 pontos): redução quantitativa e/ou mudanças nos padrões da alimentação entre os adultos da família;

IV- Insegurança grave (10 a 14 pontos): redução quantitativa e/ou mudanças nos padrões da alimentação entre as crianças da família e a fome ${ }^{3,8}$.

Para avaliar as condições socioeconômicas, foram consideradas as variáveis relacionadas à família: renda familiar, situação conjugal, número de moradores do domicílio, participação em programas de transferência de renda e classificação socioeconômica de acordo com a proposta da Associação Brasileira de Empresas de Pesquisa (ABEP) ${ }^{9}$.

A classificação da $A B E P^{9}$ considera aspectos como escolaridade, posse de bens, empregada mensalista e acesso a serviços públicos (água encanada e pavimentação das ruas). De acordo com a pontuação, que pode variar entre 0 e 100 pontos, é possível fazer a distribuição das classes em: A, B1, B2, C1, C2 e D-E

A coleta de dados antropométricos das crianças e adolescentes contemplou as variáveis idade, sexo, peso e estatura. As medidas foram aferidas com o auxílio de uma balança digital portátil Tramontina ${ }^{\circledR}$ com capacidade de 200 kg e um estadiômetro móvel Sanny ${ }^{\circledR}$ com medição de $115 \mathrm{~cm}$ a $210 \mathrm{~cm}$ para a estatura. O protocolo de medidas seguiu a Norma Técnica do Sistema de Vigilância Alimentar e Nutricional (SISVAN) ${ }^{10}$.

Para o diagnóstico do estado nutricional, foram utilizados o Índice de Massa Corporal (IMC) para idade e o índice Estatura para Idade (E/I). Os indicadores do estado nutricional foram calculados em escore z, com o auxílio do software WHO Anthro Plus ${ }^{11}$, e classificados conforme a Organização Mundial de Saúde (OMS) para crianças e adolescentes (entre 5 e 10 anos e maiores de 10 anos), de acordo com o sexo e a faixa etária ${ }^{10}$.

Os dados foram tabulados no software office Excel 2013 e posteriormente analisados no software SPSS (Statistical Package for the Social Sciences), versão 18.0. Foi utilizada a estatística descritiva simples (média, desvio padrão e percentual), e, para verificar a diferença entre os grupos (com e sem IA), foi realizado o teste qui-quadrado. Foram consideradas as diferenças significativas quando as associações obtiveram $p<0,05$.

A pesquisa foi aprovada pelo Comitê de Ética em Pesquisa (CEP) da Universidade Federal de Santa Maria (CAAE: 60302316.1.0000.5346). Os participantes assinaram o Termo de Consentimento Livre e Esclarecido e o Termo de Assentimento.

\section{RESULTADOS}

Foram avaliadas 157 crianças e adolescentes com idade entre 5 e 16 anos, pertencentes a 121 famílias rurais. A idade média dos pais ou responsáveis foi de $39 \pm 9$ anos. A maior parte das famílias estudadas morava em domicílios com 4 pessoas $(42,2 \%, n=51), 57 \%(n=69)$ tinham renda mensal de até 1 salário mínimo e $47,9 \%(n=58)$ dos pais ou responsáveis possuíam ensino fundamental incompleto. Em relação aos serviços públicos, foi observado que, em 100\% das famílias, a água do domicílio era proveniente de poço ou nascente e, em 15,7\% ( $n=19)$ das residências, o trecho do domicílio era asfaltado ou pavimentado. A classe socioeconômica D-E foi a mais predominante $(58,7 \%, n=71)$, e $46,3 \%(n=56)$ eram titulares no PBF (Tabela 1). 
Tabela 1. Distribuição das famílias segundo as características socioeconômicas e os níveis de insegurança alimentar. Palmeira das Missões, Rio Grande do Sul, Brasil, 2016 ( $n=121$ famílias)

\begin{tabular}{|c|c|c|}
\hline Variáveis & $\mathbf{n}$ & $\%$ \\
\hline \multicolumn{3}{|l|}{$N^{\circ}$ moradores na residência } \\
\hline Até 2 & 5 & 4,1 \\
\hline 3 & 39 & 32,2 \\
\hline 4 & 51 & 42,2 \\
\hline 5 ou mais & 26 & 21,5 \\
\hline \multicolumn{3}{|l|}{ Renda } \\
\hline Até 1 salário mínimo & 69 & 57,0 \\
\hline 1 a 3 & 37 & 30,6 \\
\hline 4 ou mais & 15 & 12,4 \\
\hline \multicolumn{3}{|l|}{ Escolaridade } \\
\hline Analfabeto & 22 & 18,2 \\
\hline Fundamental incompleto & 58 & 47,9 \\
\hline Fundamental completo / médio incompleto & 19 & 15,7 \\
\hline Médio completo / superior incompleto & 20 & 16,5 \\
\hline Superior completo & 2 & 1,7 \\
\hline \multicolumn{3}{|l|}{ Água } \\
\hline Poço ou nascente & 121 & 100 \\
\hline \multicolumn{3}{|l|}{ Trecho do domicílio } \\
\hline Asfaltado / pavimentado & 19 & 15,7 \\
\hline Terra / cascalho & 102 & 84,3 \\
\hline \multicolumn{3}{|l|}{ Classe socioeconômica } \\
\hline B1 & 4 & 3,3 \\
\hline B2 & 7 & 5,8 \\
\hline $\mathrm{C} 1$ & 11 & 9,1 \\
\hline $\mathrm{C} 2$ & 28 & 23,3 \\
\hline D-E & 71 & 58,7 \\
\hline \multicolumn{3}{|l|}{ Bolsa Família } \\
\hline Sim & 56 & 46,3 \\
\hline Não & 65 & 53,7 \\
\hline \multicolumn{3}{|l|}{ Níveis de insegurança alimentar } \\
\hline Segurança alimentar & 51 & 42,1 \\
\hline IA leve & 56 & 46,3 \\
\hline IA moderada & 11 & 9,1 \\
\hline IA grave & 3 & 2,5 \\
\hline
\end{tabular}

IA: insegurança alimentar 
A prevalência de $I A$, somando todos os níveis, nos domicílios avaliados com crianças e adolescentes foi de $57,9 \%(n=70)$, com maior frequência para IA leve $(46,3 \%, n=56)$, conforme a Tabela 1.

$\mathrm{Na}$ Tabela 2, estão as respostas das famílias de acordo com as questões da EBIA. As questões 1 (tiveram a preocupação de que os alimentos acabassem antes de poderem comprar mais

Tabela 2. Dados gerais de insegurança alimentar segundo as respostas das famílias rurais. Palmeira das Missões, Rio Grande do Sul, Brasil, $2016(n=121)$

\begin{tabular}{|c|c|c|}
\hline ITENS & SIM n (\%) & NÃO n (\%) \\
\hline $\begin{array}{l}\text { 1. Nos últimos três meses, os moradores deste domicílio tiveram a } \\
\text { preocupação de que os alimentos acabassem antes de poderem } \\
\text { comprar mais comida? }\end{array}$ & $57(47,1)$ & $64(52,9)$ \\
\hline $\begin{array}{l}\text { 2. Nos últimos três meses, os alimentos acabaram antes que os } \\
\text { moradores deste domicílio tivessem dinheiro para comprar mais } \\
\text { comida? }\end{array}$ & $18(14,9)$ & $103(85,1)$ \\
\hline $\begin{array}{l}\text { 3. Nos últimos três meses, os moradores deste domicílio ficaram sem } \\
\text { dinheiro para ter uma alimentação saudável e variada? }\end{array}$ & $55(45,5)$ & $66(54,5)$ \\
\hline $\begin{array}{l}\text { 4. Nos últimos três meses, os moradores deste domicílio comeram } \\
\text { apenas alguns alimentos que ainda tinham porque o dinheiro acabou? }\end{array}$ & $41(33,9)$ & $80(66,1)$ \\
\hline $\begin{array}{l}\text { 5. Nos últimos três meses, algum morador de } 18 \text { anos ou mais de } \\
\text { idade deixou de fazer alguma refeição porque não havia dinheiro para } \\
\text { comprar a comida? }\end{array}$ & $9(7,4)$ & $112(92,6)$ \\
\hline $\begin{array}{l}\text { 6. Nos últimos três meses, algum morador de } 18 \text { anos ou mais de } \\
\text { idade, alguma vez, comeu menos do que achou que devia porque não } \\
\text { havia dinheiro para comprar comida? }\end{array}$ & $10(8,3)$ & $111(91,7)$ \\
\hline $\begin{array}{l}\text { 7. Nos últimos três meses, algum morador de } 18 \text { anos ou mais de } \\
\text { idade, alguma vez, sentiu fome, mas não comeu porque não havia } \\
\text { dinheiro para comprar comida? }\end{array}$ & $3(2,5)$ & $118(97,5)$ \\
\hline $\begin{array}{l}\text { 8. Nos últimos três meses, algum morador de } 18 \text { anos ou mais de } \\
\text { idade, alguma vez, fez apenas uma refeição ao dia ou ficou um dia } \\
\text { inteiro sem comer porque não tinha dinheiro para comprar comida? }\end{array}$ & $4(3,3)$ & $117(96,3)$ \\
\hline $\begin{array}{l}\text { 9. Nos últimos três meses, algum morador com menos de } 18 \text { anos de } \\
\text { idade, alguma vez, deixou de ter uma alimentação saudável e variada } \\
\text { porque não havia dinheiro para comprar comida? }\end{array}$ & $46(38,0)$ & $75(62,0)$ \\
\hline $\begin{array}{l}\text { 10. Nos últimos três meses, algum morador com menos de } 18 \text { anos } \\
\text { de idade, alguma vez, não comeu quantidade suficiente de comida } \\
\text { porque não havia dinheiro para comprar comida? }\end{array}$ & $15(12,4)$ & $106(87,6)$ \\
\hline $\begin{array}{l}\text { 11. Nos últimos três meses, alguma vez, foi diminuída a quantidade de } \\
\text { alimentos das refeições de algum morador com menos de } 18 \text { anos de } \\
\text { idade porque não havia dinheiro para comprar comida? }\end{array}$ & $14(11,6)$ & $107(88,4)$ \\
\hline $\begin{array}{l}\text { 12. Nos últimos três meses, alguma vez, algum morador com menos } \\
\text { de } 18 \text { anos de idade deixou de fazer alguma refeição porque não havia } \\
\text { dinheiro para comprar a comida? }\end{array}$ & $5(4,1)$ & $11(95,9)$ \\
\hline $\begin{array}{l}\text { 13. Nos últimos três meses, alguma vez, algum morador com menos } \\
\text { de } 18 \text { anos de idade sentiu fome, mas não comeu, porque não havia } \\
\text { dinheiro para comprar comida? }\end{array}$ & $4(3,3)$ & $117(96,4)$ \\
\hline $\begin{array}{l}\text { 14. Nos últimos três meses, alguma vez, algum morador com menos } \\
\text { de } 18 \text { anos de idade fez apenas uma refeição ao dia ou ficou sem } \\
\text { comer por um dia inteiro porque não havia dinheiro para comprar } \\
\text { comida? }\end{array}$ & $2(1,7)$ & $119(98,3)$ \\
\hline
\end{tabular}


comida -47,1\%), 3 (ficaram sem dinheiro para ter uma alimentação saudável e variada - 45,5\%), 4 (comeram apenas alguns alimentos que ainda tinham porque o dinheiro acabou - 33,9\%) e 9 (algum morador com menos de 18 anos de idade, alguma vez, deixou de ter uma alimentação saudável e variada porque não havia dinheiro para comprar comida - 38\%) foram as que apresentaram maior proporção de respostas "sim", o que reflete a preocupação e a qualidade da alimentação da família. Já as questões $7(97,5 \%, n=118), 8(96,3 \%, n=117), 13(96,4 \%$, $n=117)$ e $14(98,3 \%, n=119)$, relacionadas à falta de alimento ou a mudanças e restrição do padrão alimentar no domicílio, obtiveram respostas negativas em maior proporção.

Quanto ao estado nutricional, foram avaliados 157 escolares (com idade entre 5 e 16 anos): 61 crianças (de 5 a 10 anos) e 96 adolescentes (acima de 10 anos a 19). Desses escolares, 44,6\% $(n=70)$ eram do sexo feminino, e $55,4 \%(n=87)$, do sexo masculino. Foi constatado que $60,7 \%$ $(n=37)$ das crianças e $57,3 \%(n=55)$ dos adolescentes estavam eutróficos. Em relação à obesidade, os percentuais encontrados foram de $19,7 \%(n=12)$ para crianças e $22,9 \%(n=22)$ para os adolescentes. Todas as crianças e a maioria $(96,9 \%, n=90)$ dos adolescentes estavam em adequação ao indicador El (Tabela 3). Não foi encontrada associação significativa entre estado nutricional e IA familiar quando o número total de crianças e adolescentes foi analisado e também estratificado de acordo com a faixa etária (dados não apresentados em tabela).

De acordo com a Tabela 4, foi observado que as famílias de classe socioeconômica mais baixa ( $D$-E) apresentaram maior prevalência de IA em comparação com as famílias pertencentes às classes A-B-C $(p<0,0001)$. Quando avaliada a relação entre a IA e a família ser beneficiária do PBF, foi verificado que as famílias participantes do programa apresentavam maiores prevalência de IA ( $p<0,0001$ ) (Tabela 5). Para termos estatísticos, nas Tabelas 4 e 5 , todos

Tabela 3. Estado nutricional das crianças e dos adolescentes. Palmeira das Missões, Rio Grande do Sul, Brasil, $2016(n=157)$

\begin{tabular}{lccc} 
& Crianças $\mathbf{n}(\%)$ & Adolescentes $\mathbf{~ ( \% ) ~}$ & Total $\mathbf{n}(\%)$ \\
\hline IMC / Idade & & & \\
\hline Magreza & $1(1,6)$ & $2(2,1)$ & $3(1,9)$ \\
\hline Eutrofia & $37(60,7)$ & $55(57,3)$ & $92(58,6)$ \\
\hline Sobrepeso & $11(18)$ & $17(17,7)$ & $28(17,8)$ \\
\hline Obesidade & $12(19,7)$ & $22(22,9)$ & $34(21,6)$ \\
\hline Total & $61(100,0)$ & $96(100,0)$ & $157(100,0)$ \\
\hline Estatura para idade & $0(0,0)$ & $6(6,3)$ & $151(96,9)$ \\
\hline Baixa estatura & $61(100,0)$ & $90(93,9)$ & $157(100,0)$ \\
\hline Estatura adequada & $61(100,0)$ & $96(100,0)$ & \\
\hline Total & & &
\end{tabular}

IMC: Índice de Massa Corporal

Tabela 4. Classe socioeconômica familiar de acordo com os níveis de insegurança alimentar. Palmeira das Missões, Rio Grande do Sul, Brasil, 2016 ( $n=121)$

\begin{tabular}{lcccc} 
& SA $\mathbf{n}(\%)$ & IA $\mathbf{n}(\%)$ & Total $\mathbf{n}$ (\%) & P* \\
\hline Classe socioeconômica & & & & $<0,000$ \\
\hline A-B-C & $32(64,0)$ & $18(36,0)$ & $50(100)$ & \\
\hline D-E & $19(26,8)$ & $52(73,2)$ & $71(100)$ & \\
\hline TOTAL & $51(42,1)$ & $70(57,9)$ & $121(100)$ & \\
\hline
\end{tabular}

SA: segurança alimentar; IA: insegurança alimentar; $P^{*}$ : teste qui-quadrado 
Tabela 5. Insegurança alimentar entre as famílias beneficiadas pelo Programa Bolsa Família. Palmeira das Missões, Rio Grande do Sul, Brasil, $2016(n=121)$

\begin{tabular}{lllll} 
& SA $\mathbf{n}(\%)$ & IA $\mathbf{n}(\%)$ & Total & P* \\
\hline Bolsa Família & & & & \\
\hline Sim & $09(16,1)$ & $47(83,9)$ & $56(100)$ & $<0,0001$ \\
\hline Não & $42(64,6)$ & $23(35,4)$ & $65(100)$ & \\
\hline
\end{tabular}

SA: segurança alimentar; IA: insegurança alimentar; $\mathrm{P}^{*}$ : teste qui-quadrado

os participantes que apresentaram algum nível de IA, fosse leve, moderada ou grave, foram classificados na categoria "Insegurança Alimentar" e, em relação a condição socioeconômica, todos os participantes foram divididos em duas classes: alta a média $=A-B-C ;$ e baixa $=D-E$.

\section{DISCUSSÃO}

A população rural tem sido estudada por ser um grupo mais vulnerável às condições de $I A$, as quais se relacionam com baixa renda e escolaridade, dificuldade de acesso a alimentos e presença de moradores com menos de 18 anos no domicílio ${ }^{3,5}$. Neste estudo, foram avaliadas famílias rurais com crianças e adolescentes e foi constatada alta prevalência de IA nelas. A maior frequência foi na forma leve, condição relacionada às características socioeconômicas.

Quando os resultados do presente estudo são comparados com os dados nacionais ${ }^{3}$, é observado que a prevalência de IA encontrada $(57,9 \%)$ foi o dobro da média para todo o território nacional (22,6\%). Uma possível explicação para esse resultado é o fato de a amostra estudada ser exclusivamente de famílias rurais, que são mais vulneráveis às condições de IA. Ainda com base na PNAD, os dados de IA deste estudo $(57,9 \%)$ estão acima da média para a região Sul $(14,9 \%)$ e para o Rio Grande do Sul (15,9\%).

Prevalências superiores de IA (55\% a 88\%) também estão presentes em outros estudos que avaliaram populações rurais ${ }^{4,5,12}$. Vale destacar que todos esses estudos foram realizados na região Nordeste do país, onde se concentram elevados níveis de pobreza da população ${ }^{13}$. Esses autores encontraram relação entre a IA e os menores estratos socioeconômicos, assim como neste estudo. No trabalho de Ferreira et al. ${ }^{12}$, a IA também se associou aos domicílios com menores de 18 anos, confirmando que essas famílias apresentam maiores riscos para IA.

Estudo realizado com a população urbana no Rio Grande do Sul, em São Leopoldo ${ }^{14}$, encontrou prevalência de IA menor que este estudo $(41,1 \%)$. Embora Palmeira das Missões e São Leopoldo possuam Índice de Desenvolvimento Humano $(\mathrm{IDH})^{7}$ semelhantes, o município do presente estudo, diferentemente de São Leopoldo, é caracterizado pela produção agrícola em monocultura (cerca de $62 \%$ do território se destina à monocultura da soja) ${ }^{15}$. Historicamente, a tradição latifundiária relaciona-se com o baixo desenvolvimento e a pobreza no rural ${ }^{16}$, possibilitando uma possível justificativa para um valor mais alto de IA encontrado neste estudo que avaliou apenas a população rural.

Neste estudo, as famílias participantes do PBF apresentaram alta prevalência de IA em relação às não participantes. Outras pesquisas ${ }^{5,12}$ corroboram esses achados, em que os níveis de IA aumentaram diretamente com a proporção das famílias beneficiadas por programas sociais, principalmente aquelas com IA grave.

De modo geral, as famílias beneficiadas pelo PBF pertencem às classes sociais mais baixas, e isso evidencia que o programa atende efetivamente as famílias que se encontram em maior vulnerabilidade ${ }^{12}$. Traldi et al. ${ }^{17}$ constataram que as famílias em condições de IA beneficiadas pelo PBF utilizavam os recursos financeiros recebidos, em maior parte, para a aquisição de alimentos.

O PBF tem se mostrado de extrema importância para as famílias no que diz respeito à diminuição da pobreza e evolução das condições de segurança alimentar e nutricional ${ }^{18}$. Para as famílias rurais, o PBF pode ser uma forma de complementar a renda, principalmente em 
circunstâncias em que o acesso a outra forma de remuneração seja escasso ou a produção na propriedade para o consumo próprio não forneça subsídios suficientes para garantir uma alimentação adequada.

Outro resultado importante neste estudo foi a alta prevalência de obesidade nas crianças e adolescentes quando comparada com os dados nacionais para a mesma faixa etária. De acordo com a Pesquisa de Orçamentos Familiares (POF) ${ }^{19}$, a proporção de obesidade costuma ser inferior em áreas rurais (7,5\% em crianças e 2,8\% nos adolescentes). Neste estudo, os resultados superaram os achados da POF para as áreas rural e urbana. Outros autores também encontraram altas prevalências de sobrepeso/obesidade em crianças $^{20}$ e adolescentes ${ }^{21}$ rurais. Destaca-se também que a obesidade é encontrada com maior frequência em crianças $^{19}$, mas, no presente estudo, os adolescentes apresentaram maior proporção.

O quadro epidemiológico encontrado nas crianças e adolescentes deste estudo demonstra que a prevalência de excesso de peso e obesidade foi alta, inclusive em classes socioeconômicas mais baixas, enquanto o déficit de peso e estatura foi pouco frequente.

Embora encontrada alta prevalência de sobrepeso e obesidade nas crianças e adolescentes, não foi observada relação com a IA familiar. Da mesma forma, outros estudos ${ }^{20,22-24}$ realizados em âmbito rural e urbano também não encontraram essa relação, mesmo que houvesse a coexistência de IA e excesso de peso no contexto familiar.

Os efeitos da IA podem manifestar-se de diferentes formas, desde a desnutrição até o excesso de peso ${ }^{25}$. O estado nutricional é uma condição multifatorial que pode ser ocasionada pela falta do alimento, mas também por hábitos alimentares inadequados, condições socioeconômicas e de saúde ${ }^{2}$. Para crianças e adolescentes, a disponibilidade do alimento no domicílio é importante para manter uma alimentação adequada, entretanto fatores relacionados aos responsáveis pela aquisição dos alimentos, como a renda e a escolaridade, são decisivos para boas escolhas alimentares ${ }^{26}$.

Em famílias de baixo poder aquisitivo, o consumo alimentar pode estar fundamentado em escolhas alimentares mais baratas e de baixo valor nutritivo ${ }^{27}$. Esse padrão alimentar pode explicar a prevalência de sobrepeso e obesidade encontrada em famílias socialmente vulneráveis. De acordo com o estudo de Ruschel et al. ${ }^{14}$, indivíduos em situação de IA, principalmente crianças, apresentaram maiores probabilidades de consumo inadequado, como alto consumo de biscoitos, salgadinhos, refrigerantes e sucos artificiais, que vão ao encontro do baixo consumo de alimentos in natura, como frutas e verduras.

Uma limitação deste estudo foi a não realização da avaliação do consumo alimentar das famílias, das crianças e dos adolescentes, o que impossibilita a análise da qualidade da alimentação na relação do estado nutricional e destinação da verba do PBF para este fim (compra de alimentos).

Destaca-se que o contexto estudado foi a zona rural de um município do interior do Rio Grande do Sul, caracterizado pela produção predominante do cultivo de soja. Nessa localidade, não há políticas públicas que incentivem, promovam e garantam a segurança alimentar e nutricional da população, em especial no meio rural, onde permanecem dificuldades de acesso à saúde, educação e alimentação adequada.

A partir disso, é importante a elaboração de ações intersetoriais com o objetivo de criar fontes de renda e trabalho para as famílias rurais e promover a saúde nesse meio. Nesse contexto, destacam-se o Programa de Aquisição de Alimentos (PAA ${ }^{28}$, o Programa de Saúde na Escola (PSE) ${ }^{29}$ e o Programa Nacional de Alimentação Escolar (PNAE) ${ }^{30}$. Para isso, é necessária a articulação entre gestores municipais, entidades de assistência técnica (saúde e educação) e agricultores familiares.

Políticas públicas, como o $\mathrm{PAA}^{28}$ e o $\mathrm{PNAE}^{30}$, podem contribuir para o acesso a alimentos saudáveis e de qualidade mediante a compra de alimentos produzidos no município, por exemplo. Outra importante estratégia que pode ser adotada, por meio do PSE, é a realização de ações de educação alimentar e nutricional no ambiente escolar, as quais poderiam envolver crianças, adolescentes e seus pais e familiares para orientação e incentivo a escolhas alimentares saudáveis. 
As famílias avaliadas apresentaram alta prevalência de IA relacionada inversamente com as condições de renda e classe socioeconômica e a inclusão em políticas de transferência de renda, como o PBF. O estado nutricional das crianças e adolescentes não apresentou relação com a IA familiar. É necessário que haja ações intersetoriais de promoção das políticas públicas, bem como regulação dos seus propósitos para melhorar as condições de segurança alimentar e nutricional das famílias rurais, visto que o PBF tem um importante papel no consumo alimentar das famílias.

\section{REFERÊNCIAS}

1. Brasil. Lei no 11.346 de 15 de setembro de 2006. Lei Orgânica de Segurança Alimentar e Nutricional. Diário Oficial da União [Internet], Brasília, 18 de setembro de 2006. https://www.planalto.gov.br/ ccivil_03/_ato2004-2006/2006/lei/l11346.htm

2. Food and Agriculture Organization. $O$ estado da segurança alimentar e nutricional no Brasil: um retrato multidimensional. Brasília; 2014.

3. Instituto Brasileiro de Geografia e Estatística. Pesquisa nacional por amostras de domicílios: segurança alimentar 2013. Rio de Janeiro: IBGE; 2014.

4. Aires JS, Martins MC, Joventino ES, Ximenes LB. (In) Segurança alimentar em famílias de pré-escolares de uma zona rural do Ceará. Acta Paul Enferm. 2012;25(1):102-8. http://dx.doi.org/10.1590/S010321002012000100018.

5. Vianna RPT, Segall-Corrêa AM. Insegurança alimentar das famílias residentes em municípios do interior do estado da Paraíba, Brasil. Rev Nutr. 2008;21(Supl):111-22. http://dx.doi.org/10.1590/S141552732008000700010

6. Rocha NP, Milagres LC, Novaes JF, Franceschini SCC. Association between food and nutrition insecurity with cardiometabolic risk factors in childhood and adolescence: a systematic review. Rev Paul Pediatr. 2016;34(2):225-33. http://dx.doi.org/10.1016/j.rpped.2015.08.007. PMid:26564327.

7. Instituto Brasileiro de Geografia e Estatística - IBGE. Dados estatísticos provenientes do censo demográfico. Rio de Janeiro: IBGE; 2010.

8. Segall-Corrêa AM, Marin-León L, Melgar-Quinonez H, Pérez-Escamilla R. Refinement of the Brazilian Household Food Insecurity Measurement Scale: Recommendation for a 14-item EBIA. Rev Nutr. 2014;27(2):241-51. http://dx.doi.org/10.1590/1415-52732014000200010.

9. Associação Brasileira de Empresas de Pesquisa. Critério de classificação econômica Brasil. São Paulo: ABEP; 2015.

10. Brasil. Ministério da Saúde. Orientações para a coleta e análise de dados antropométricos em serviços de saúde. Norma técnica do Sistema de Vigilância Alimentar e Nutricional - SISVAN. Brasília; 2011.

11. World Health Organization. WHO Anthro Plus. Personal Computers: software for assessing growth of the word's children and adolescents [software]. Geneva; 2010.

12. Ferreira HC, Souza MECA, Moura FA, Horta BL. Prevalência e fatores associados à Insegurança Alimentar e Nutricional em famílias dos municípios do norte de Alagoas, Brasil, 2010. Cien Saude Colet. 2014;19(5):153342. http://dx.doi.org/10.1590/1413-81232014195.06122013. PMid:24897218.

13. Soares S, Souza L, Silva W, Silveira FG, Campos A. Perfil da pobreza: Norte e Nordeste rurais. Brasília; 2016.

14. Ruschel LF, Henn RL, Backes V, Melo P, Marques LAS, Olinto MTA. Insegurança alimentar e consumo alimentar inadequado em escolares da rede municipal de São Leopoldo, RS, Brasil. Cien Saude Colet. 2016;21(7):2275-86. http://dx.doi.org/10.1590/1413-81232015217.00742015. PMid:27383360.

15. Deepask. Soja: veja produção agrícola e área plantada por cidade do Brasil - Palmeira das Missões, RS [Internet]. 2017 [citado em 2017 jan 18]. Disponível em: http://www.deepask.com/goes?page=palmeiradas-missoes/RS-Soja:-Veja-a-producao-agricola-e-a-area-plantada-no-seu-municipio

16. OXFAM Brasil. Terrenos da desigualdade: terra, agricultura e desigualdades no Brasil rural. São Paulo; 2016 [citado em 2017 jan 25]. Disponível em: http://www.fao.org/family-farming/detail/es/c/459287/

17. Traldi DR, Almeida LMMC, Ferrante VLSB. Repercussões do Programa Bolsa Família no município de Araraquara, SP: um olhar sobre a segurança alimentar e nutricional dos beneficiários. Interações. 2013;13(1):23-37.

18. Kopruszynski CP, Costa VMH. Programas de transferência condicionada de renda e segurança alimentar e nutricional. Segur Aliment Nutr. 2016;23:996-1007. http://dx.doi.org/10.20396/san.v23i0.8635618. 
19. Instituto Brasileiro de Geografia e Estatística. Pesquisa de orçamentos familiares: antropometria e análise do estado nutricional de crianças e adolescentes e adultos no Brasil. Rio de Janeiro: IBGE; 2010.

20. Jackson JA, Smit E, Manore MM, John D, Gunter K. The family-home nutrition environment end dietary intake in rural children. Nutrients. 2015;7(12):9707-20. http://dx.doi.org/10.3390/nu7125495. PMid:26610566.

21. Barros MS, Fonseca VM, Meio MDBB, Chaves CR. Excesso de peso entre adolescentes em zona rural e a alimentação escolar oferecida. Cad Saude Colet. 2013;21(2):201-8. http://dx.doi.org/10.1590/S1414462X2013000200016.

22. Guerra LD, Espinosa MM, Bezerra ACD, Guimarães LV, Lima-Lopes MP. Insegurança alimentar em domicílios com adolescentes da Amazônia Legal Brasileira: prevalência e fatores associados. Cad Saude Publica. 2013;29(2):335-48. http://dx.doi.org/10.1590/S0102-311X2013000200020. PMid:23459819.

23. Gundersen C, Lohman BJ, Eisenmann JC, Garasky S, Stewart SD. Child-specific food insecurity and overweight are not associated in a sample of 10- to 15-year-old low-income youth. J Nutr. 2008;138(2):3718. http://dx.doi.org/10.1093/jn/138.2.371. PMid:18203906.

24. Oliveira JS, Lira PIC, Veras ICL, Maia SR, Lemos MCC, Andrade SLLS, et al. Estado nutricional e insegurança alimentar de adolescentes e adultos em duas localidades de baixo índice de desenvolvimento humano. Rev Nutr. 2009;22(4):453-65. http://dx.doi.org/10.1590/S1415-52732009000400002.

25. Frongillo EA. Confronting myths about household food insecurity and excess weight. Cad Saude Publica. 2013;29(2):229-41. http://dx.doi.org/10.1590/S0102-311X2013000200005. PMid:23459804.

26. Estima CCP, Philippi ST, Alvarenga MS. Fatores determinantes de consumo alimentar: por que os indivíduos comem o que comem? Rev Bras Nutr Clín. 2009;4(24):263-8.

27. Drewnowski A. Obesity, diets and social inequality. Nutr Rev. 2009;67:S36-9. http://dx.doi.org/10.1111/ j.1753-4887.2009.00157.x.

28. Brasil. Ministério do Desenvolvimento Agrário. Fome zero: a experiência brasileira. Brasília; 2010.

29. Brasil. Decreto n 6.286 , de 5 de dezembro de 2007. Institui o Programa Saúde na Escola - PSE, e dá outras providências. Diário Oficial da União [Internet], Brasília, 6 de dezembro de 2007.

30. Brasil. Resolução/CD/FNDE n²6, de 17 de junho de 2013. Dispõe sobre o atendimento da alimentação escolar aos alunos da educação básica no âmbito do Programa Nacional de Alimentação Escolar - PNAE. Diário Oficial da União [Internet], Brasília, 18 de junho de 2013. 